\title{
On the Robust Stability of Continuous-Time and Discrete-Time Time-Invariant Uncertain Systems with Rational Dependence on the Uncertainty: a Non-Conservative Condition
}

\author{
Graziano Chesi
}

\begin{abstract}
A key problem in automatic control consists of investigating robust stability of systems with uncertainty. This paper considers linear systems with rational dependence on time-invariant uncertainties constrained in the simplex. It is shown that a sufficient condition for establishing whether the system is either stable or unstable can be obtained by solving a generalized eigenvalue problem constructed through homogeneous parameter-dependent quadratic Lyapunov functions (HPD-QLFs). Moreover, it is shown that this condition is also necessary for establishing either stability or instability by using a sufficiently large degree of the HPD-QLF. Some numerical examples illustrate the use of the proposed approach in both cases of continuous-time and discrete-time uncertain systems.
\end{abstract}

\section{INTRODUCTION}

It is well-known that stability of linear systems with time-invariant uncertainty constrained in a polytope is an important problem in automatic control. Various methods have been proposed for addressing this problem, generally exploiting parameter-dependent Lyapunov functions and linear matrix inequalities (LMIs). For instance, Lyapunov functions with linear dependence are exploited in [15], Lyapunov functions with polynomial dependence are considered in [1], and homogeneous parameter-dependent quadratic Lyapunov functions (HPD-QLFs) are proposed in [11]. Parameterdependent Lyapunov functions are considered also in [20] which proposes a general framework for LMI relaxations, in [18] where homogeneous solutions are characterized, in [16] which addresses the case of semi-algebraic sets, in [17], [19] where matrix-dilation approaches are considered, and in [14] which exploits $D / G$-scaling in the case of rational dependence on the uncertainty.

Some of these methods provide necessary and sufficient conditions for robust stability. However, the necessity is achieved for an unknown degree of the polynomials used. This implies that, if the system is unstable, no conclusion can be reached. This paper addresses this problem via HPD-QLFs for in the case of rational dependence on the uncertainty. It is shown that a sufficient condition for establishing either stability or instability can be obtained by solving a generalized eigenvalue problem (GEVP), which belongs to the class of quasi-convex optimization problems. Moreover, it is shown that this condition is also necessary for establishing either stability or instability by using a sufficiently large degree of the HPD-QLF. The idea behind

G. Chesi is with the Department of Electrical and Electronic Engineering, University of Hong Kong, Pokfulam Road, Hong Kong, China. Contact information: see http://www. eee.hku.hk/ ${ }^{\sim}$ chesi this condition is to exploit the LMI relaxation introduced in [9], [11] via the square matricial representation (SMR) in order to characterize the instability via the presence of suitable vectors in certain eigenspaces. The SMR allows one to establish if a polynomial (or a matrix polynomial) is a sum of squares (SOS) of polynomials via an LMI, see e.g. the pioneering works [13], [9] and the recent works [12], [3]. Some numerical examples illustrate the use of the proposed approach in both cases of continuous-time and discrete-time uncertain systems. This paper re-elaborates and extends the results proposed in [7].

The paper is organized as follows. Section II introduces the problem formulation and the SMR. Section III describes the proposed approach. Section IV reports some numerical examples. Lastly, Section V concludes the paper with some final remarks.

\section{Preliminaries}

\section{A. Problem Formulation}

Notation:

- $\mathbb{N}, \mathbb{R}, \mathbb{C}$ : natural, real and complex numbers;

- $\mathbb{R}_{0}: \mathbb{R} \backslash\{0\}$

- $I_{n}: n \times n$ identity matrix;

- $A>0$ : symmetric positive definite matrix;

- $A \otimes B$ : Kronecker's product;

- $A^{\prime}, \operatorname{tr}(A), \operatorname{det}(A):$ transpose, trace and determinant of $A$;

- $\operatorname{spc}(A)=\{\lambda \in \mathbb{C}: \operatorname{det}(\lambda I-A)=0\}$

- $\operatorname{span}\left(v_{1}, \ldots, v_{k}\right)=\left\{a_{1} v_{1}+\ldots+a_{k} v_{k}, a_{1}, \ldots, a_{k} \in\right.$ $\mathbb{R}\}$;

- $\operatorname{sq}(p)=\left(p_{1}^{2}, \ldots, p_{q}^{2}\right)^{\prime}$ with $p \in \mathbb{R}^{q}$

- $\partial y(p)$ : degree of the polynomial $y(p)$;

- CT, DT: continuous-time and discrete-time;

- s.t.: subject to.

Let us consider the uncertain system

$$
\left\{\begin{array}{ll}
(\text { CT case) } & \dot{x}(t)=A(p) x(t) \\
\text { (DT case) } & x(t+1)=A(p) x(t)
\end{array} \quad \forall p \in \mathcal{P}\right.
$$

where $t \in \mathbb{R}$ is the time, $x(t) \in \mathbb{R}^{n}$ is the state, $p \in \mathbb{R}^{q}$ is the uncertain parameter, and $\mathcal{P}$ is the simplex defined as

$$
\mathcal{P}=\left\{p \in \mathbb{R}^{q}: \sum_{i=1}^{q} p_{i}=1, \quad p_{i} \geq 0\right\} .
$$

The function $A: \mathbb{R}^{q} \rightarrow \mathbb{R}^{n \times n}$ is a matrix rational function, i.e. a matrix whose entries are rational functions. In particular 
we express $A(p)$ as follows:

$$
A(p)=\frac{1}{b(p)}\left(\begin{array}{ccc}
a_{1,1}(p) & \ldots & a_{1, n}(p) \\
\vdots & \ddots & \vdots \\
a_{n, 1}(p) & \ldots & a_{n, n}(p)
\end{array}\right)
$$

where all the generic entry $a_{i, j}(p)$ with $i, j=1, \ldots, n$ and $b(p)$ are polynomials in $p$.

Let us define the set of matrices

$$
\mathcal{A}=\left\{A(p) \in \mathbb{R}^{n \times n}: p \in \mathcal{P}\right\} .
$$

In the sequel we will say that:

- (CT case) $A(p)$ is stable if and only if $\operatorname{Re}(\lambda)<0$ for all $\lambda \in \operatorname{spc}(A(p))$;

- (DT case) $A(p)$ is stable if and only if $|\lambda|<1$ for all $\lambda \in \operatorname{spc}(A(p))$;

- $\mathcal{A}$ is stable if and only if $A(p)$ is stable for all $p \in \mathcal{P}$, i.e. whenever the following condition holds:

$$
\operatorname{Re}(\lambda)<0 \quad \forall \lambda \in \operatorname{spc}(A(p)) \forall p \in \mathcal{P} ;
$$

- $\mathcal{A}$ (resp., $A(p)$ ) is unstable if $\mathcal{A}$ (resp., $A(p)$ ) is not stable. Hence, $\mathcal{A}$ is unstable whenever the following condition holds:

$$
\exists p \in \mathcal{P}, \lambda \in \operatorname{spc}(A(p)): \operatorname{Re}(\lambda) \geq 0 .
$$

The aim of this paper is to allows one to establish whether $\mathcal{A}$ is either stable or unstable, hence providing a solution for this decidability problem.

\section{B. $S M R$}

Before proceeding we briefly introduce a key tool that will be exploited in the next sections to derive the proposed conditions. For $p \in \mathbb{R}^{n}$, let $y(p)$ be a homogeneous polynomial of degree $2 d$, i.e. a polynomial with only monomials of degree $2 d$ :

$$
y(p)=\sum_{\substack{i_{1}+\ldots+i_{n}=2 d \\ i_{1} \geq 0, \ldots, i_{q} \geq 0}} c_{i_{1}, \ldots, i_{q}} p_{1}^{i_{1}} \cdots p_{q}^{i_{q}} .
$$

Let $p^{\{d\}} \in \mathbb{R}^{\sigma(q, d)}$ be a vector containing all monomials of degree equal to $d$ in $p$, where $\sigma(q, d)$ is the number of such monomials given by

$$
\sigma(q, d)=\frac{(q+d-1) !}{(q-1) ! d !}
$$

Then, $y(p)$ can be expressed via the square matrix representation (SMR) introduced in [13] as

$$
y(p)=p^{\{d\}^{\prime}}(Y+L(\alpha)) p^{\{d\}}
$$

where $Y=Y^{\prime} \in \mathbb{R}^{\sigma(p, d) \times \sigma(p, d)}$ is a symmetric matrix such that

$$
y(p)=p^{\{d\}^{\prime}} Y p^{\{d\}},
$$

$L(\alpha)=L(\alpha)^{\prime} \in \mathbb{R}^{\sigma(p, d) \times \sigma(p, d)}$ is a linear parametrization of the set

$$
\mathcal{L}(d)=\left\{L=L^{\prime}: p^{\{d\}^{\prime}} L p^{\{d\}}=0\right\}
$$

and $\alpha \in \mathbb{R}^{\sigma_{\mathcal{L}}}$ is a vector of free parameters, where $\sigma_{\mathcal{L}}$ is the dimension of the linear subspace $\mathcal{L}(d)$ given by

$$
\sigma_{\mathcal{L}}=\frac{1}{2} \sigma(q, d)(\sigma(q, d)+1)-\sigma(q, 2 d) .
$$

The matrices $P$ and $P+L(\alpha)$ are referred to as SMR matrix and complete SMR matrix, respectively, of $p(x)$. The matrix $P$ is also known as Gram matrix of $p(x)$.

The SMR is useful because it allows one to investigate positivity of polynomials. Indeed, one can establish whether a polynomial is a sum of squares of polynomials (SOS) by solving a convex optimization problem with linear matrix inequalities (LMIs) as proposed in [13]. Specifically, $y(p)$ is SOS if and only if there exists $\alpha$ such that

$$
Y+L(\alpha) \geq 0
$$

which is an LMI feasibility test since $Y$ is constant and $L(\alpha)$ is a linear matrix function. LMI feasibility tests can be checked by solving a convex optimization problem, see for instance [2].

The SMR allows one to represent also matrix homogeneous polynomials. Specifically, let us introduce the notation

$$
\Delta(z, Z)=\left(z \otimes I_{n}\right)^{\prime} Z\left(z \otimes I_{n}\right)
$$

where $z$ is a vector and $Z=Z^{\prime}$ is a symmetric matrix of suitable dimension. Let $Y(p)=Y(p)^{\prime}$ be a symmetric matrix homogeneous polynomial of degree $2 d$. Then, $Y(p)$ can be expressed via the SMR as done in [9] as

$$
Y(p)=\Delta\left(p^{\{d\}}, Z+U(\alpha)\right)
$$

where $Z=Z^{\prime} \in \mathbb{R}^{n \sigma(p, d) \times n \sigma(p, d)}$ is a symmetric matrix such that

$$
Y(p)=\Delta\left(p^{\{d\}}, Z\right)
$$

$U(\alpha)=U(\alpha)^{\prime} \in \mathbb{R}^{\sigma(p, d) \times \sigma(p, d)}$ is a linear parametrization of the set

$$
\mathcal{U}(d)=\left\{U=U^{\prime}: \Delta\left(p^{\{d\}}, U\right)=0\right\},
$$

and $\alpha \in \mathbb{R}^{\sigma \mathcal{U}}$ is a vector of free parameters, where $\sigma_{\mathcal{U}}$ is the dimension of the linear subspace $\mathcal{U}(d)$ given by

$$
\sigma_{\mathcal{U}}=\frac{1}{2} n(\sigma(q, d)(n \sigma(q, d)+1)-(n+1) \sigma(q, 2 d)) .
$$

The matrices $Z$ and $Z+U(\alpha)$ are referred to as SMR matrix and complete SMR matrix, respectively, of $Y(p)$.

As in the scalar case, the SMR allows one to establish whether $Y(p)$ is SOS, i.e. there exist matrix homogeneous polynomials $Y_{1}(p), \ldots, Y_{k}(p)$ of suitable dimension and degree such that

$$
Y(p)=\sum_{i=1}^{k} Y_{i}(p)^{\prime} Y_{i}(p) .
$$

Indeed, $Y(p)$ is SOS if and only if there exists $\alpha$ such that [9]

$$
Z+U(\alpha) \geq 0
$$

which is an LMI feasibility test. 
The reader is also referred to [10], [11], [5], [12] for details and algorithms on the SMR and its use for detecting SOS polynomials. See also the survey paper [3].

\section{Proposed Results}

\section{A. Reformulation via Matrix Homogeneous Polynomials}

In this section we provide an equivalent formulation of the problem described in Section II-A by using matrix homogeneous polynomials.

First of all, let us observe that, in order for $\mathcal{A}$ to be stable and bounded, the polynomial $b(p)$ in (3) can be supposed to satisfy

$$
b(p)>0 \quad \forall p \in \mathcal{P}
$$

without loss of generality.

Let us rewrite $A(p)$ as

$$
A(p)=\frac{1}{b(p)} \sum_{i=1}^{m_{A}} A_{i}(p)
$$

where $m_{A}$ is the maximum of the degrees of the numerators in $A(p)$, i.e.

$$
m_{A}=\max _{\substack{i=1, \ldots, n \\ j=1, \ldots, n}} \partial a_{i, j}(p)
$$

and $A_{i}(p) \in \mathbb{R}^{n \times n}$ is a matrix polynomial of degree $i$ in $p$. We define the new function

$$
M(p)=\sum_{i=1}^{m_{A}} A_{i}(p)\left(\sum_{j=1}^{q} p_{j}\right)^{m_{A}-i} .
$$

It follows that $M(p) \in \mathbb{R}^{n \times n}$ is a matrix homogeneous polynomial of degree $m_{A}$. Moreover, it can be verified that

$$
A(p)=\frac{1}{b(p)} M(p) \quad \forall p \in \mathcal{P} .
$$

Taking into account the condition (21), it follows that

$$
\mathcal{A} \text { is stable } \Longleftrightarrow \mathcal{M} \text { is stable }
$$

where

$$
\mathcal{M}=\left\{M(p) \in \mathbb{R}^{n \times n}: p \in \mathcal{P}\right\} .
$$

\section{B. $H P D-Q L F S$}

In order to introduce the proposed result, let us briefly recall the definition of homogeneous parameter-dependent quadratic Lyapunov functions (HPD-QLFs) and the stability result proposed in [9], [11], [6], [12].

For $m \in \mathbb{N}$ let us define the linear subspace

$\mathcal{S}(m)=\left\{S=S^{\prime}: \Delta\left(p^{\{m\}}, S\right)\right.$ does not contain

monomials $p_{1}^{i_{1}} \ldots p_{q}^{i_{q}}$ with at least one $i_{j}$ odd $\}$.

Let $S(\beta)=S(\beta)^{\prime} \in \mathbb{R}^{n \sigma(p, m) \times n \sigma(p, m)}$ be a linear parametrization of $\mathcal{S}(m)$, and let us define the matrix function

$$
P(\operatorname{sq}(p), \beta)=\Delta\left(p^{\{m\}}, S(\beta)\right) .
$$

The candidate HPD-QLFs of degree $m$ can be written as

$$
v(x, p)=x^{\prime} P(p, \beta) x
$$

for some $\beta$.

Let us define the integer

$$
\begin{array}{ll}
\text { (CT case) } & d=m_{A} \\
\text { (DT case) } & d=2 m_{A}
\end{array}
$$

and the matrix function

$$
\begin{aligned}
& \text { (CT case) } Q(\operatorname{sq}(p), \beta)=-B^{\prime} C-C B \\
& \text { (DT case) } Q(\operatorname{sq}(p), \beta)=\left(\sum_{i=1}^{q} p_{i}^{2}\right)^{d} C-B^{\prime} C B
\end{aligned}
$$

for $B=M(\mathrm{sq}(p))$ and $C=P(\mathrm{sq}(p), \beta)$.

Let $R(\beta)$ be a SMR matrix of the matrix form $Q(\operatorname{sq}(p), \beta)$, i.e. a symmetric function satisfying

$$
\Delta\left(p^{\{m+d\}}, R(\beta)\right)=Q(\mathrm{sq}(p), \beta)
$$

and let $U(\alpha)=U(\alpha)^{\prime} \in \mathbb{R}^{n \sigma(p, m+d) \times n \sigma(p, m+d)}$ be a linear parametrization of the set $\mathcal{U}(m+d)$ defined in (17).

The following theorem provides is given in [11], [6], [12] and investigates stability of the set $\mathcal{M}$ in (27) via HPD-QLFs.

Theorem 1: [11], [6], [12] The set $\mathcal{M}$ in (27) is stable if and only if there exists $m$ such that the following LMIs hold for some $\alpha, \beta$ :

$$
\left\{\begin{array}{l}
S(\beta)>0 \\
R(\beta)+U(\alpha)>0
\end{array}\right.
$$

\section{Establishing Stability and Instability}

Let us define

$$
T(\beta)=\Delta\left(K, I_{d q} \otimes S(\beta)\right)
$$

where $K$ is the matrix satisfying

$$
\underbrace{p \otimes \cdots \otimes p}_{d \text { times }} \otimes p^{\{m\}}=K p^{\{m+d\}} .
$$

We have that

$$
\Delta\left(p^{\{m+d\}}, T(\beta)\right)=\left(\sum_{i=1}^{q} p_{i}^{2}\right)^{d} P(\operatorname{sq}(p), \beta) .
$$

Let us define the optimization problem

$$
\eta^{*}=\sup _{\alpha, \beta, \eta} \eta \text { s.t. }\left\{\begin{array}{l}
S(\beta)>0 \\
R(\beta)+U(\alpha)-\eta T(\beta)>0 \\
\operatorname{tr}(S(\beta))=1 .
\end{array}\right.
$$

Let $\alpha^{*}, \beta^{*}$ be optimal values of $\alpha, \beta$ in (38), and let us define

$$
V=R\left(\beta^{*}\right)+U\left(\alpha^{*}\right) .
$$

Let $c_{1}, \ldots, c_{r}$ be the eigenvectors of the non-positive eigenvalues of $V$, i.e.

$$
\left\{\begin{array}{l}
c_{i}^{\prime} c_{i}=1 \\
V c_{i}=\lambda_{i} c_{i} \text { for some } \lambda_{i} \in \mathbb{R}, \lambda_{i} \leq 0 .
\end{array}\right.
$$

The following result provides a necessary and sufficient condition for establishing whether $\mathcal{A}$ is either stable or unstable.

Theorem 2: The set $\mathcal{A}$ is stable if and only if there exists $m$ such that $\eta^{*}>0$. Moreover, $\mathcal{A}$ is unstable if and only 
if there exist $m$ and $(u, y) \in \mathbb{R}_{0}^{q} \times \mathbb{R}_{0}^{n}$ such that $A(\xi(u))$ is unstable and

$$
u^{\{m+d\}} \otimes y \in \operatorname{span}\left\{c_{1}, \ldots, c_{r}\right\}
$$

where $\xi: \mathbb{R}_{0}^{q} \rightarrow \mathcal{P}$ is the function

$$
\xi(u)=\left(\sum_{i=1}^{q} u_{i}^{2}\right)^{-1} \mathrm{sq}(u) .
$$

Proof. Let us consider the stability statement, and let us observe that $K$ in (36) is a full column rank (see e.g. [11]), which directly implies from (35) that

$$
T(\beta)>0 \Longleftrightarrow S(\beta)>0 .
$$

Therefore, from (43) and Theorem 1 one has that $\mathcal{M}$ is stable if and only if there exists $m$ such that $\eta^{*}>0$. Indeed, let us observe that the constraint $\operatorname{tr}(S(\beta))=1$ is not restrictive since $S(\beta), R(\beta), U(\alpha$ and $T(\beta)$ are linear functions, and it is introduced in order to normalized the solution of (38). Then, since (26) holds, we conclude that $\mathcal{A}$ is stable if and only if there exists $m$ such that $\eta^{*}>0$.

Let us consider the instability statement. The sufficiency is obvious because, if $A(\xi(u))$ is unstable and $\xi(u) \in \mathcal{P}$, then $\mathcal{A}$ is unstable for definition. Hence, let us consider the necessity and let us assume that $\mathcal{A}$ is unstable. From the stability statement we have $\eta^{*} \leq 0$, and from (26) we have that also $\mathcal{M}$ is unstable. Let us suppose for contradiction that, for all $m$, (41) does not hold.

Let us consider firstly the CT case. This supposition implies that $\operatorname{Re}(\lambda)<-0.5 \eta^{*}$ for all $\lambda \in \operatorname{spc}(M(p))$ for all $p \in \mathcal{P}$. In fact, from (29)-(33), (37) and Lemma 3 in [11], the first two constraints in (38) imply

$$
\left\{\begin{array}{l}
P(p)>0 \\
Q(p)-\eta P(p)>0
\end{array} \quad \forall p \in \mathcal{P} .\right.
$$

Consequently, there exists $\varepsilon>0$ such that $M(p)+0.5\left(\eta^{*}+\right.$ $\varepsilon) I$ is stable for all $p \in \mathcal{P}$. Let us replace $M(p)$ with $M(p)+$ $0.5\left(\eta^{*}+\varepsilon\right) I$ in our original problem. It follows that the new set $\mathcal{M}$ is stable, and the new solution of (38), which we refer to as $\eta^{\#}$, satisfies $\eta^{\#}=-\varepsilon$. But since $\varepsilon>0$ this implies that (34) is not satisfied for any $m$, hence contradicting Theorem 1.

Let us consider now the DT case. The supposition that (41) does not hold for any $m$ implies that $|\lambda|<\sqrt{1-\eta^{*}}$ for all $\lambda \in \operatorname{spc}(M(p))$ for all $p \in \mathcal{P}$. Consequently, one has that there exists $\varepsilon \in(0,1)$ such that $M(p)\left(\varepsilon \sqrt{1-\eta^{*}}\right)^{-1}$ is stable for all $p \in \mathcal{P}$. Let us replace $M(p)$ with $M(p)\left(\varepsilon \sqrt{1-\eta^{*}}\right)^{-1}$ in our original problem. It follows that the new set $\mathcal{M}$ is stable, and the new solution of (38), which we refer to as $\eta^{\#}$, satisfies $\eta^{\#}=1-\varepsilon^{-2}$. But since $\varepsilon \in(0,1)$ this implies that (34) is not satisfied for any $m$, contradicting again Theorem 1.

Theorem 2 requires to solve (38), which is a generalized eigenvalue problem (GEVP) and hence a quasi-convex optimization problem [2]. The pairs $(u, y)$ in $\mathbb{R}_{0}^{q} \times \mathbb{R}_{0}^{n}$ satisfying (41) can be found with the technique in [8], [4], [12] which amounts to finding the roots of a polynomial obtained via pivoting. The vectors $c_{1}, \ldots, c_{r}$ can be obtained once (38) has been solved, being eigenvectors of $V$.

In order to clarify the construction of (38), let us consider a simple situation with $n=2, q=2, m_{A}=1$ and $m=0$ in the CT case. We can select $p^{\{m\}}=1$ and $p^{\{m+d\}}=$ $\left(p_{1}, p_{2}\right)^{\prime}$. A parametrization $S(\beta)$ for the set $\mathcal{S}$ in (28), the matrix $K$ in (36) and the matrix $T(\beta)$ in (35) are hence

$$
\begin{gathered}
S(\beta)=\left(\begin{array}{cc}
\beta_{1} & \beta_{2} \\
\star & \beta_{3}
\end{array}\right) \\
K=I_{2} \\
T(\beta)=I_{2} \otimes S(\beta) .
\end{gathered}
$$

Let us consider another situation with $n=2, q=2, m_{A}=1$ and $m=1$ in the CT case. We can select $p^{\{m\}}=\left(p_{1}, p_{2}\right)^{\prime}$ and $p^{\{m+d\}}=\left(p_{1}^{2}, p_{1} p_{2}, p_{2}^{2}\right)^{\prime}$. A parametrization $S(\beta)$, the matrix $K$ and the matrix $T(\beta)$ are hence

$$
\begin{gathered}
S(\beta)=\left(\begin{array}{cccc}
\beta_{1} & \beta_{2} & 0 & -\beta_{7} \\
\star & \beta_{3} & \beta_{7} & 0 \\
\star & \star & \beta_{4} & \beta_{5} \\
\star & \star & \star & \beta_{6}
\end{array}\right) \\
K=\left(\begin{array}{ccc}
1 & 0 & 0 \\
0 & 1 & 0 \\
0 & 1 & 0 \\
0 & 0 & 1
\end{array}\right) \\
T(\beta)=K^{\prime}\left(I_{2} \otimes S(\beta)\right) K .
\end{gathered}
$$

The matrices $R(\beta)$ and $U(\alpha)$ can be computed with simple algorithms, see for instance [12] for details.

Let us conclude this section by remarking that the proposed condition provides a solution for the decidability problem of establishing whether $A(p)$ is either stable or unstable. Indeed, for any chosen degree $m$ of the HPDQLF, Theorem 2 allows one to establish either stability (if $\eta^{*}>0$ ) or instability (if (41) holds). Moreover, the theorem guarantees that, for a finite $m$, one of these conditions is satisfied.

\section{EXAMPLES}

Here we present some illustrative examples of the proposed approach.

\section{A. Example 1}

Let us consider in the CT case the uncertain system

$$
\dot{x}=G(\theta) x
$$

where

$$
G(\theta)=\left(\begin{array}{ccc}
-0.5 & 0 & 1.5 \theta-0.5 \\
0 & -3 & 2 \\
1-\theta & 2-1.5 \theta & -1
\end{array}\right)
$$

and $\theta \in \mathbb{R}$ is an uncertain parameter satisfying

$$
\theta \in[0,1] .
$$

Let us define

$$
p=(\theta, 1-\theta)^{\prime} .
$$


We have that $n=3, q=2, b(p)=1, m_{A}=1$ and

$M(p)=\left(\begin{array}{ccc}-0.5 p_{1}-0.5 p_{2} & 0 & p_{1}-0.5 p_{2} \\ 0 & -3 p_{1}-3 p_{2} & 2 p_{1}+2 p_{2} \\ p_{2} & 0.5 p_{1}+2 p_{2} & -p_{1}-p_{2}\end{array}\right)$.

Let us use $m=1$. We find that the solution of (38) satisfies $\eta^{*}<0$. Then, Theorem 2 proves that $\mathcal{A}$ is unstable, in particular we obtain

$$
\begin{aligned}
\xi(u) & =(0.4336,0.5664)^{\prime} \\
\operatorname{spc}(A(\xi(u))) & =\{0.0436,-0.6142,-3.9293\} .
\end{aligned}
$$

Some details are: $\beta \in \mathbb{R}^{14} ; \alpha \in \mathbb{R}^{15} ; u$ is found from (41) with the technique in [8], [12] by finding the roots of a quadratic polynomial.

\section{B. Example 2}

Let us consider (1) in the DT case with $A(p)$ given by

$$
\begin{aligned}
& A_{1,1}(p)=-0.2 p_{1}+0.4 p_{2}-0.2 p_{3} \\
& A_{1,2}(p)=0.6 p_{1}-0.3 p_{2}+1.6 p_{3} \\
& A_{1,3}(p)=0.1 p_{1}+0.3 p_{2}+1.4 p_{3} \\
& A_{2,1}(p)=p_{1}-0.7 p_{2}-0.7 p_{3} \\
& A_{2,2}(p)=0.4 p_{1}-0.5 p_{2}+0.8 p_{3} \\
& A_{2,3}(p)=-0.4 p_{1}+0.7 p_{2}-0.1 p_{3} \\
& A_{3,1}(p)=1.3 p_{1}-0.7 p_{2}+0.6 p_{3} \\
& A_{3,2}(p)=0.1 p_{1}-1.7 p_{2}-1.1 p_{3} \\
& A_{3,3}(p)=0.4 p_{1}+1.4 p_{2}-0.4 p_{3} .
\end{aligned}
$$

Hence, we have $n=3, q=3, b(p)=1, m_{A}=1$ and $M(p)=A(p)$. Let us use $m=0$. We find that the solution of (38) satisfies $\eta^{*}<0$. Then, Theorem 2 proves that $\mathcal{A}$ is unstable, in particular we obtain

$$
\begin{aligned}
\xi(u) & =(0.4443,0.0000,0.5557)^{\prime} \\
\operatorname{spc}(A(\xi(u))) & =\{-1.322,0.7549 \pm 0.2254\} .
\end{aligned}
$$

Some details are: $\beta \in \mathbb{R}^{5} ; \alpha \in \mathbb{R}^{81} ; u$ is found from (41) with the technique in [8], [12] by finding the roots of a quadratic polynomial.

\section{Example 3}

Let us consider in the CT case the uncertain system

$$
\dot{x}=G(\theta) x
$$

where

$$
G(\theta)=G_{0}+G_{1}(\theta)+G_{2}(\theta)
$$

with

$$
\begin{gathered}
G_{0}=\left(\begin{array}{ccc}
-1 & 0 & 0 \\
0 & -2 & 0 \\
3 & 0 & -1
\end{array}\right) \\
G_{1}(\theta)=\left(\begin{array}{ccc}
0 & 0 & \theta_{1} \\
0 & \theta_{1} & -\theta_{2} \\
0 & 0 & 1
\end{array}\right) \\
G_{2}(\theta)=\left(\begin{array}{ccc}
-3 \theta_{1}^{2} & 0 & 0 \\
0 & 0 & 0 \\
0 & -\theta_{1} \theta_{2} & -2 \theta_{2}^{2}
\end{array}\right)
\end{gathered}
$$

and $\theta=\left(\theta_{1}, \theta_{2}\right)^{\prime} \in \mathbb{R}^{2}$ is an uncertain parameter satisfying

$$
\theta_{1}+\theta_{2} \leq 1, \quad \theta_{i} \geq 0 \forall i=1,2 .
$$

Let us define

$$
p=\left(\theta_{1}, \theta_{2}, 1-\theta_{1}-\theta_{2}\right)^{\prime} .
$$

We have $n=3, q=3, b(p)=1, m_{A}=2$ and

$$
\begin{aligned}
& M_{1,1}(p)=-4 p_{1}^{2}-2 p_{1} p_{2}-2 p_{1} p_{3}-p_{2}^{2}-2 p_{2} p_{3}-p_{3}^{2} \\
& M_{1,2}(p)=0 \\
& M_{1,3}(p)=p_{1}^{2}+p_{1} p_{2}+p_{1} p_{3} \\
& M_{2,1}(p)=0 \\
& M_{2,2}(p)=-p_{1}^{2}-3 p_{1} p_{2}-3 p_{1} p_{3}-2 p_{2}^{2}-4 p_{2} p_{3}-2 p_{3}^{2} \\
& M_{2,3}(p)=-p_{1} p_{2}-p_{2}^{2}-p_{2} p_{3} \\
& M_{3,1}(p)=3 p_{1}^{2}+6 p_{1} p_{2}+6 p_{1} p_{3}+3 p_{2}^{2}+6 p_{2} p_{3}+6 p_{3}^{2} \\
& M_{3,2}(p)=-p_{1} p_{2} \\
& M_{3,3}(p)=-p_{1}^{2}-p_{1} p_{2}-2 p_{1} p_{3}-2 p_{2}^{2}-p_{2} p_{3}-p_{3}^{2} .
\end{aligned}
$$

Let us use $m=0$. We find that the solution of (38) satisfies $\eta^{*}<0$. Then, Theorem 2 proves that $\mathcal{A}$ is unstable, in particular we obtain

$$
\begin{aligned}
\xi(u) & =(0.5959,0.3263,0.0779)^{\prime} \\
\operatorname{spc}(A(\xi(u))) & =\{0.0167,-1.4232,-2.9494\} .
\end{aligned}
$$

Some details are: $\beta \in \mathbb{R}^{5} ; \alpha \in \mathbb{R}^{81} ; u$ is found from (41) with the technique in [8], [12] by finding the roots of a quadratic polynomial.

Next, we change the entry $(1,3)$ of $G_{1}(\theta)$ from $\theta_{1}$ to $-\theta_{1}$ and repeat the investigation. With $m=0$ we find $\eta^{*}>0$, hence implying from Theorem 2 that $\mathcal{A}$ is stable.

\section{Example 4}

Let us consider in the CT case the uncertain system

$$
\dot{x}=G(\theta) x
$$

where

$$
G(\theta)=\left(\begin{array}{ccc}
-1-4 \theta_{2} & 0 & 1-2 \theta_{1}-2 \theta_{2} \\
\theta_{2} & -5-\theta_{2} & 4-4 \theta_{2} \\
2 \theta_{1} & 2+2 \theta_{1} & -2
\end{array}\right)
$$

and $\theta=\left(\theta_{1}, \theta_{2}\right)^{\prime} \in \mathbb{R}^{2}$ is an uncertain parameter satisfying

$$
\theta \in[0,1]^{2} .
$$

Let us define

$$
A(p)=\sum_{i=1}^{4} p_{i} G\left(\theta^{(i)}\right)
$$

where $p=\left(p_{1}, \ldots, p_{4}\right)^{\prime} \in \mathcal{P}$ and

$$
\begin{aligned}
& \theta^{(1)}=\left(\begin{array}{l}
0 \\
0
\end{array}\right), \quad \theta^{(2)}=\left(\begin{array}{l}
1 \\
0
\end{array}\right) \\
& \theta^{(3)}=\left(\begin{array}{l}
0 \\
1
\end{array}\right), \quad \theta^{(4)}=\left(\begin{array}{l}
1 \\
1
\end{array}\right) .
\end{aligned}
$$


We have $n=3, q=4, b(p)=1, m_{A}=1$ and

$$
\begin{aligned}
& M_{1,1}(p)=-p_{1}-p_{2}-5 p_{3}-5 p_{4} \\
& M_{1,2}(p)=0 \\
& M_{1,3}(p)=p_{1}-p_{2}-p_{3}-3 p_{4} \\
& M_{2,1}(p)=p_{3}+p_{4} \\
& M_{2,2}(p)=-5 p_{1}-5 p_{2}-6 p_{3}-6 p_{4} \\
& M_{2,3}(p)=4 p_{1}+4 p_{2} \\
& M_{3,1}(p)=2 p_{2}+2 p_{4} \\
& M_{3,2}(p)=2 p_{1}+4 p_{2}+2 p_{3}+4 p_{4} \\
& M_{3,3}(p)=-2 p_{1}-2 p_{2}-2 p_{3}-2 p_{4} .
\end{aligned}
$$

Let us use $m=1$. We find that the solution of (38) satisfies $\eta^{*}<0$. Then, Theorem 2 proves that $\mathcal{A}$ is unstable, in particular we obtain

$$
\begin{aligned}
\xi(u) & =(0.6300,0.3562,0.0010,0.0128)^{\prime} \\
\operatorname{spc}(A(\xi(u))) & =\{0.2072,-1.1456,-7.1305\} .
\end{aligned}
$$

Some details are: $\beta \in \mathbb{R}^{41} ; \alpha \in \mathbb{R}^{255} ; u$ is found from (41) with the technique in [8], [12] by finding the roots of a quadratic polynomial.

\section{CONCLUSION}

This paper has proposed a necessary and sufficient condition for establishing either stability or instability of linear systems with rational dependence on uncertainties constrained in the simplex. This condition is built by using HPDQLFs, and amounts to solving a GEVP, which is a quasiconvex optimization problem. Some numerical examples have illustrated the proposed approach in both cases of continuous-time and discrete-time uncertain systems.

\section{REFERENCES}

[1] P.-A. Bliman. A convex approach to robust stability for linear systems with uncertain scalar parameters. SIAM Journal on Control and Optimization, 42(6):2016-2042, 2004.

[2] S. Boyd, L. El Ghaoui, E. Feron, and V. Balakrishnan. Linear Matrix Inequalities in System and Control Theory. SIAM, 1994.

[3] G. Chesi. LMI techniques for optimization over polynomials in control: a survey. IEEE Trans. on Automatic Control, to appear.

[4] G. Chesi. Establishing tightness in robust H-infinity analysis via homogeneous parameter-dependent Lyapunov functions. Automatica, 43(11):1992-1995, 2007.

[5] G. Chesi. On the gap between positive polynomials and SOS of polynomials. IEEE Trans. on Automatic Control, 52(6):1066-1072, 2007.

[6] G. Chesi. On the non-conservatism of a novel LMI relaxation for robust analysis of polytopic systems. Automatica, 44(11):2973-2976, 2008.

[7] G. Chesi. Time-invariant uncertain systems: a necessary and sufficient condition for stability and instability via HPD-QLFs. Automatica, 46(2):471-474, 2010.

[8] G. Chesi, A. Garulli, A. Tesi, and A. Vicino. Characterizing the solution set of polynomial systems in terms of homogeneous forms: an LMI approach. Int. Journal of Robust and Nonlinear Control, 13(13):1239-1257, 2003.

[9] G. Chesi, A. Garulli, A. Tesi, and A. Vicino. Robust stability for polytopic systems via polynomially parameter-dependent Lyapunov functions. In IEEE Conf. on Decision and Control, pages 4670-4675, Maui, Hawaii, 2003.

[10] G. Chesi, A. Garulli, A. Tesi, and A. Vicino. Solving quadratic distance problems: an LMI-based approach. IEEE Trans. on Automatic Control, 48(2):200-212, 2003.

[11] G. Chesi, A. Garulli, A. Tesi, and A. Vicino. Polynomially parameterdependent Lyapunov functions for robust stability of polytopic systems: an LMI approach. IEEE Trans. on Automatic Control, 50(3):365-370, 2005.
[12] G. Chesi, A. Garulli, A. Tesi, and A. Vicino. Homogeneous Polynomial Forms for Robustness Analysis of Uncertain Systems. Springer, 2009.

[13] G. Chesi, A. Tesi, A. Vicino, and R. Genesio. On convexification of some minimum distance problems. In European Control Conf., Karlsruhe, Germany, 1999.

[14] Y. Ebihara, Y. Onishi, and T. Hagiwara. Robust performance analysis of uncertain LTI systems: Dual LMI approach and verifications for exactness. IEEE Trans. on Automatic Control, 54(5):938-951, 2009.

[15] E. N. Goncalves, R. M. Palhares, R. H. C. Takahashi, and R. C. Mesquita. New approach to robust d-stability analysis of linear timeinvariant systems with polytope-bounded uncertainty. IEEE Trans. on Automatic Control, 51(10):1709-1714, 2006.

[16] J. Lavaei and A. G. Aghdam. Robust stability of LTI systems over semi-algebraic sets using sum-of-squares matrix polynomials. IEEE Trans. on Automatic Control, 53(1):417-423, 2008.

[17] Y. Oishi. An asymptotically exact approach to robust semidefinite programming problems with function variables. IEEE Trans. on Automatic Control, 54(5):1000-1006, 2009.

[18] R. C. L. F. Oliveira and P. L. D. Peres. Parameter-dependent LMIs in robust analysis: Characterization of homogeneous polynomially parameter-dependent solutions via LMI relaxations. IEEE Trans. on Automatic Control, 52(7):1334-1340, 2007.

[19] D. Peaucelle and M. Sato. LMI tests for positive definite polynomials: Slack variable approach. IEEE Trans. on Automatic Control, 54(4):886-891, 2009.

[20] C. W. Scherer. LMI relaxations in robust control. European Journal of Control, 12(1):3-29, 2006. 